\section{INOVAÇÃO E CONTRIBUIÇÃO DO DESIGN THINKING: MAPEAMENTO BIBLIOMÉTRICO SOBRE A PRODUÇÃO ACADÊMICANAS BASES SCOPUS E WEB SCIENCE}

\author{
INNOVATION AND THE CONTRIBUTION OF \\ DESIGN THINKING: BIBLIOMETRIC MAPPING ON \\ ACADEMIC PRODUCTION IN SCOPUS AND WEB \\ SCIENCE BASES
}

Elisangela de Menezes Aragão emaragaos@hotmail.com Doutoranda em Ciência da Propriedade Intelectual pela Universidade Federal de Sergipe. São Cristóvão - SE - BR.

\section{Vania de Jesus vaniajesus2@gmail.com Doutoranda e Mestre do Programa de Pós-Graduação em Ciência da Propriedade Intelectual pela Universidade Federal de Sergipe. São Cristóvão - SE - BR.}

Ramon Santos Carvalho ramondabarra18@hotmail.com Doutor e Mestre do Programa de Pós-Graduação em Ciência da Propriedade Intelectual pela Universidade Federal de Sergipe. São Cristóvão - SE - BR.

Mario Jorge Campos dos Santos mjkampos@gmail.com Doutorado em Recursos Florestais em Conservação de Ecossistemas Florestais. Professor associado da Universidade Federal de Sergipe. São Cristóvão - SE - BR.

\section{RESUMO}

Começam a despontar, nas diversas áreas de conhecimento, diferentes tipos de inovação, oriundos de metodologias como Design Thinking, que desempenham um papel importante nas organizações, funcionando como facilitadoras na apresentação de novas soluções que possibilitam melhores resultados. Este artigo tem o objetivo de apresentar a relação entre Inovação e metodologias para o despertar criativo, alinhada ao Design Thinking, por meio da produção acadêmica no período de 2015 a 2020, utilizando a bibliometria. Para o alcance do objetivo deste estudo, utilizaram-se indicadores bibliométricos na análise e avaliação dos artigos nas bases de dados Scopus e Web of Science. Obtiveram-se, na base Scopus, 92 artigos e, na Web of Science, 83, no período de 2015 a 2020, mostrando um espaço para as pesquisas neste contexto. Destaca-se pela quantidade de artigos o ano de 2018. Observou-se, por fim, que este tema é de interesse de várias áreas de conhecimentos, apesar de a maior concentração ser nas áreas administração de negócios, engenharia, ciência da computação e ciências sociais, outras áreas também têm contribuído com artigos, caracterizando que este é um tema multidisciplinar.

Palavras-chave: design thinking; criatividade; processo de inovação.

\begin{abstract}
Different types of innovations are beginning to emerge in different areas of knowledge, arising from methodologies such as Design Thinking, which plays an important role in organizations, working as facilitators in the presentation of new solutions that enable better results. This article aims to present the relationship between
\end{abstract}


Innovation and methodologies for creative awakening, aligned to Design Thinking, through the academic production from 2015 to 2020, using bibliometrics. To achieve the objective of this study, bibliometric indicators were used in the analysis and evaluation of articles in the Scopus and Web of Science databases. In the Scopus database, 92 articles were obtained, and in the Web of Science 83, from 2015 to 2020, showing a space for research in this context. The year 2018 stands out for the number of articles. Finally, it was noted that this topic is of interest to several areas of knowledge, despite the highest concentration being in the areas of business administration, engineering, computer science, and social sciences, other areas have also contributed articles, characterizing that this is a multidisciplinary theme.

Keywords: design thinking; creativity; innovation process

\section{INTRODUÇÃO}

No início deste século, a inovação tornou-se um dos assuntos mais discutidos pelas organizações, sendo um dos fatores determinantes para o sucesso no mercado. Cada vez mais competitivo, o mercado promove uma busca por diferenciação, e as organizações precisam obter avanços na produtividade, em geral, decorrente de uma nova forma de fazer as coisas, na utilização eficiente e eficaz no uso dos recursos ou por processos completamente novos.

Buscar pela essência das coisas e ter ideias para melhorar e transformar o ambiente de negócio em um ecossistema inovador é um desafio global, de maneira que os produtos entregues hoje, rapidamente, estarão desatualizados, e equilibrar as necessidades de indivíduos e da sociedade como um todo é algo que deve ser pensado localmente, mas visando a uma atuação global.

Para alcançar um desempenho que atenda às incertezas do mercado, às quais novas práticas se fazem pertinentes, é essencial que o indivíduo, suas capacidades e competências resultem em uma nova cultura de trabalho, voltada à cooperação e sinergia entre áreas multidisciplinares, destacando a criatividade e geração de inovações. Dentro das diversas técnicas utilizadas para o despertar criativo nas inovações, o Design Thinking (DT) surge, conforme explicam Bonini e Sbragia (2011), como um modelo de alto potencial para geração de resultados diferenciados nas organizações que buscam a liderança. É uma ferramenta de trabalho centrada no indivíduo, que estimula o desenvolvimento, o compartilhamento de ideias para chegar ao novo, a soluções criativas, impactando, positivamente, a organização com o propósito de transformar desafios em oportunidades e projeto inovadores.

A jornada da inovação no ambiente econômico atual requer um aprendizado sistêmico, que pode ser obtido em vários estágios práticos e por meio de experiências pessoais, pois todos os negócios têm como base a troca de informações e conhecimento entre pessoas, e cada uma delas traz consigo experiências e aprendizagens únicas. Assim, com o foco no ser humano, o DT é um método que auxilia pessoas, por meio de um processo colaborativo, a criar soluções criativas e inovadoras para desafios complexos da nossa sociedade.

Assim, para conhecer os estudos acadêmicos produzidos nesta área, a pesquisa optou por realizar um estudo bibliométrico com abordagem quantitativa e qualitativa, e tem como objetivo apresentar a relação entre inovação e metodologias para o despertar criativo, neste caso, por meio da ferramenta Design Thinking que atua como facilitadora na geração da inovação.

\section{REFERENCIAL TEÓRICO}

\subsection{INOVAÇÃO}

Inovar sugere introduzir novidade, adotar e implementar uma nova ideia (processo, bem ou serviço) em uma organização em resposta a um problema investigado, transformando uma nova ideia em algo concreto (ALENCAR, 
1996). Para Schumpeter (1984), a inovação é o motor principal do crescimento econômico, estimulando a dinâmica do ambiente competitivo global e estimulando as empresas a buscarem vantagens competitivas e diferenciação do mercado, para obter melhores resultados econômicos.

Segundo o Manual de Oslo (OECD, 2018, p. 20):

Uma inovação é um produto ou processo novo ou aprimorado (ou combinação dos mesmos) que difere significativamente dos produtos ou processos anteriores da unidade e que foi disponibilizado para usuários potenciais (produto) ou colocado em uso pela unidade (processo). As atividades de inovação incluem todas as atividades de desenvolvimento, financeiras e comerciais realizadas por uma empresa que se destinam a resultar em uma inovação para a empresa.

A inovação pode-se apresentar de várias formas, seja no produto, marketing, processos, pessoas ou organizacional. Sendo assim, inovar é desenvolver a criatividade para implementação de um novo ou, significativamente, melhorado produto (bem ou serviço), processo de trabalho, ou prática de relacionamento entre pessoas, grupos e organizações.

As definições básicas de inovação de produto e de processo de negócio, segundo o Manual de Oslo (OECD, 2018, p. 20), são as seguintes:

Inovação de produto é um produto ou serviço novo ou aprimorado que difere significativamente dos bens ou serviços anteriores da empresa e que foram introduzidos no mercado. Inovação de processo de negócio é um processo de negócio novo ou aprimorado para uma ou mais funções de negócios que diferem significativamente dos processos de negócios anteriores e que foi colocado em uso pela empresa.
No entendimento de Sharif, Baark e Lau (2012), em uma economia globalizada e baseada no conhecimento, as organizações devem inovar continuamente. As empresas que inovam utilizam várias técnicas para inovar e expandir as fronteiras de seus processos de produção de conhecimento a fim de colaborar com organizações externas em busca de um processo de inovação contínuo. Em longo prazo, a inovação e geração de novas tecnologias são importantes para o desenvolvimento econômico, impulsionando o sucesso das organizações e sustentando vantagens competitivas.

O processo de inovação consiste em um caminho organizado para a resolução de problemas, partindo de operações sistemáticas, combinando conhecimento, habilidades e comportamento de um indivíduo (PÄRTTÖ; SAARILUOMA, 2012; VERGANTI; ÖBERG, 2013). Compreende, também, um conjunto de atividades estruturadas e padronizadas que envolvem a identificação e análise de uma oportunidade, geração e seleção de ideias e conceito, até o lançamento no mercado (HACKLIN et al., 2009; KOEN et al., 2001).

Tohidi e Jabbari (2012) apresentam uma definição complementar ao processo de inovação, definindo-o em atividades estruturadas de operações técnicas industriais e comerciais, para gerar ideias de processos, produtos ou serviços novos. Para Gordon et al. (2008), compreende as atividades e habilidades necessárias para gerar algo novo, desde a concepção até o lançamento no mercado. A compreensão da maneira como as fases do processo de inovar são desenvolvidas e a relação entre elas consistem em um elemento importante para entender como a inovação é gerada. Fatores econômicos e sociais são importantes para entender como ocorre a inovação e o papel que ela exerce.

\subsection{DESIGN THINKING}

O "design" como disciplina tem por objetivo máximo promover bem-estar na vida das pessoas. O designer enxerga como um problema tudo aquilo que prejudica ou impede a 
experiência (emocional, cognitiva, estética) e o bem-estar na vida das pessoas. Isso faz que sua principal tarefa seja identificar problemas e gerar soluções. E essa maneira como um designer observa as coisas e age sobre elas chamou a atenção de gestores, em busca por novos caminhos para a inovação empresarial (VIANNA et al., 2012).

O Design Thinking (DT) foi desenvolvido por meio da necessidade de buscar novos caminhos para a inovação, sendo uma abordagem focada no ser humano, em que a multidisciplinaridade, a colaboração e a tangibilização de pensamentos e processos conduzem a soluções inovadoras (VIANNA et al., 2012). O DT está sendo, cada vez mais, procurado por organizações que precisam ampliar seu repertório de estratégias para enfrentar desafios complexos e abertos (DORST, 2011).

Seus defensores afirmam que ele pode ajudar a resolver desafios contemporâneos em áreas tão diversas como políticas, saúde, mudanças climáticas, emprego entre outras.
O design centrado no homem ou no usuário enfatiza a compreensão das visões e experiências dos cidadãos durante os estágios da definição do problema. Assim, as ferramentas de design podem continuar sendo empregadas nos modelos de escolha racional (em vez de desafiar), tornando-se parte da metodologia de busca de alternativas durante o processo de considerar como resolver um problema (CONSIDINE, 2012).

O processo de DT é descrito por vários autores com fases distintas, contudo, basicamente, ocorre por meio de ciclos interativos de exploração (LIEDTKA, 2015). Conforme ilustrado na figura 1 , o processo possui, inicialmente, uma fase imersão, compreensão do problema e identificação das necessidades, por intermédio de coleta de dados que definirá os critérios do projeto; uma segunda fase de conceituação e geração de ideias; e uma terceira fase de prototipagem, testes e seleção das ideias desenvolvidas (BROWN; WYATT, 2010; VIANNA et al., 2012).

Figura 1 - Etapas do processo de design thinking

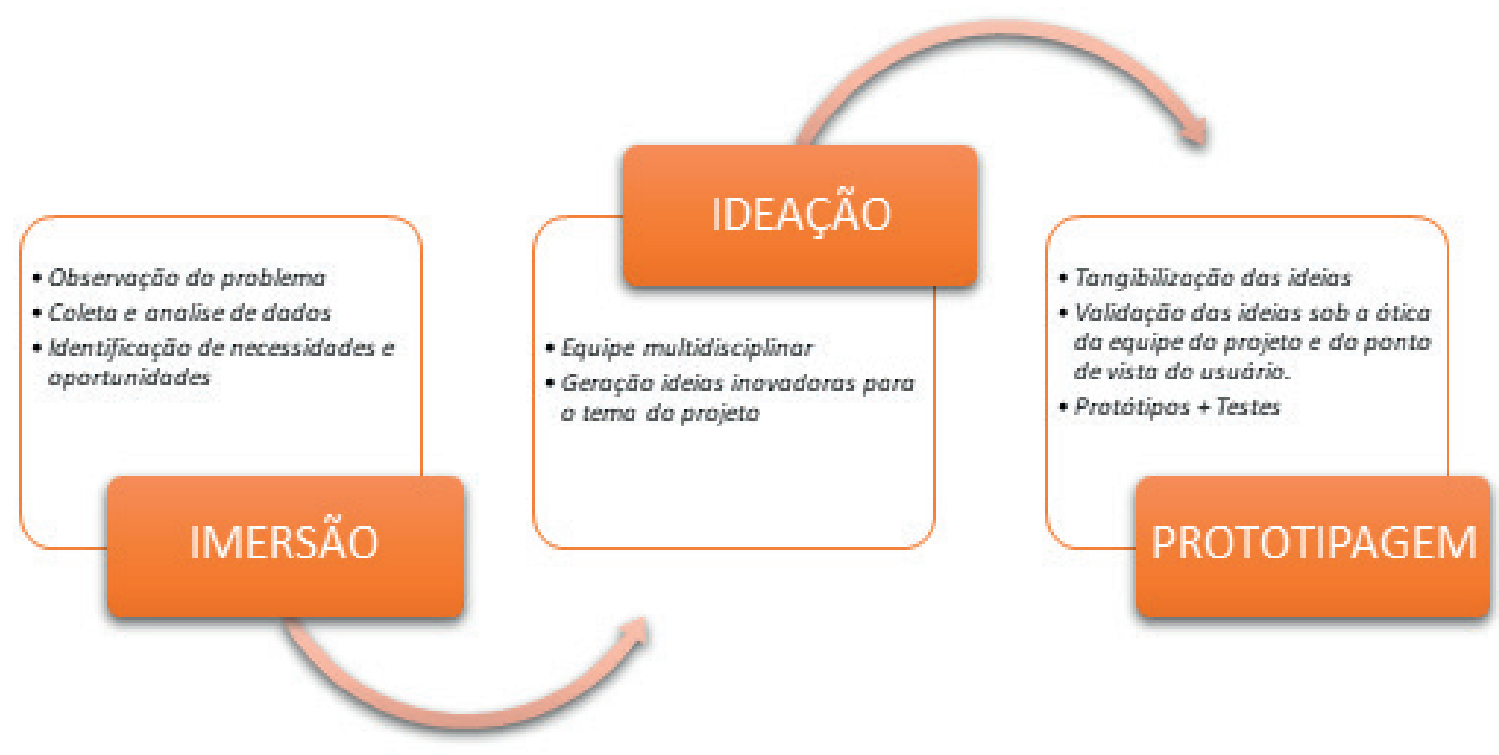

Fonte: elaborado a partir de Brown e Wyatt (2010) e Vianna et al. (2012). 
De acordo com Brown e Wyatt (2010), podem ser destacados alguns benefícios ou vantagens do uso do $D T$, como: soluções inovadoras com o foco no ser humano; velocidade na implantação de melhorias dos processos; engajamento das equipes (motivação e otimismo); desenvolvimento da autonomia; visão multidisciplinar e holística (interna e externa); incentivo ao erro e acerto; utilização de recursos visuais, desenho, diagramas de causa e efeito; uso de protótipos para validar ou gerar ideias; oferta de produtos e serviços alinhada com as exigências de mercado; persistência, criatividade e colaboração.

Considera-se o DT um processo de inovação com valor percebido pelo cliente, ou seja, é uma abordagem que tem como objetivo atingir a satisfação do cliente, seja ele interno ou externo. A satisfação está diretamente relacionada ao fato de suprir as necessidades, os desejos, as percepções e expectativas. O que atrai no DT é a agilidade, os baixos custos envolvidos no processo de geração de inovação e o valor (BROWN; WYATT, 2010). O diferencial que o método do design thinking apresenta é a maneira como seus elementos individuais são combinados, sendo um conjunto de abordagens, atitudes e ferramentas que, quando juntos, integram todas as pontas na resolução de problemas (LIEDTKA, 2015).

\section{METODOLOGIA}

Neste trabalho, foi realizada uma pesquisa bibliométrica, para uma análise com abordagem quantitativa e qualitativa da produção acadêmica publicada em periódicos, anais, livros etc., buscando relacionar a inovação com a metodologia do Design Thinking.

Foram utilizadas as bases de dados Scopus e Web of Science para o levantamento, utilizou-se como termos de busca "INNOVATION" AND "DESIGN THINKING" no subcampo "Article title" e limitado ao período de janeiro de "2015" até julho de "2020". Dessa forma, foram pesquisados os documentos que apresentavam os termos no título, refinando a busca para obtenção de documentos focados no objetivo da pesquisa.

Utilizaram-se as ferramentas de análises próprias das bases de dados para chegar a resultados obtidos por meio dos filtros disponíveis para refinar a busca. Dos diferentes documentos contidos nessas bases, para este estudo, foram considerados todos os tipos contabilizados: artigos de conferência, artigos científicos, capítulo de livro, relatórios, dentre outros. Como categorias de análise, foram considerados os seguintes indicadores: publicações por ano, tipos de documentos, países, áreas de conhecimento, principais instituições de ensino, periódicos, quantitativo de publicações por autor, principais autores.

Esses dados foram exportados no formato CSV e analisados no Excel e no software VOsviewer, uma ferramenta baseada em Java que pode ser usada para criar e explorar representações visuais de coleções de documentos, ajudando o usuário a entender o seu conteúdo. Foram analisadas as citações e cocitações, trabalhando a premissa que autores citam artigos que consideram importantes no desenvolvimento de suas pesquisas.

\section{RESULTADOS E DISCUSSÃO}

Seguindo o percurso metodológico, foram obtidos 92 documentos na base Scopus e 83 na Web of Science. Foi possível observar, de acordo com a gráfico 1, quando comparados os dados das duas bases, que, na Scopus nos anos de 2016, com 20 e 2018 com 19, evidencia-se um maior número de publicações, porém, no ano de 2020, se considerarmos que a busca foi realizada tendo como lastro final o mês de julho de 2020, a produção segue a média dos anos anteriores. Na, base Web of Science, verifica-se uma crescente de 2015 até um maior número de publicações em 2018, totalizando, naquele ano, 23, havendo uma queda nos dois anos seguintes. 
Gráfico 1 - Publicações por ano

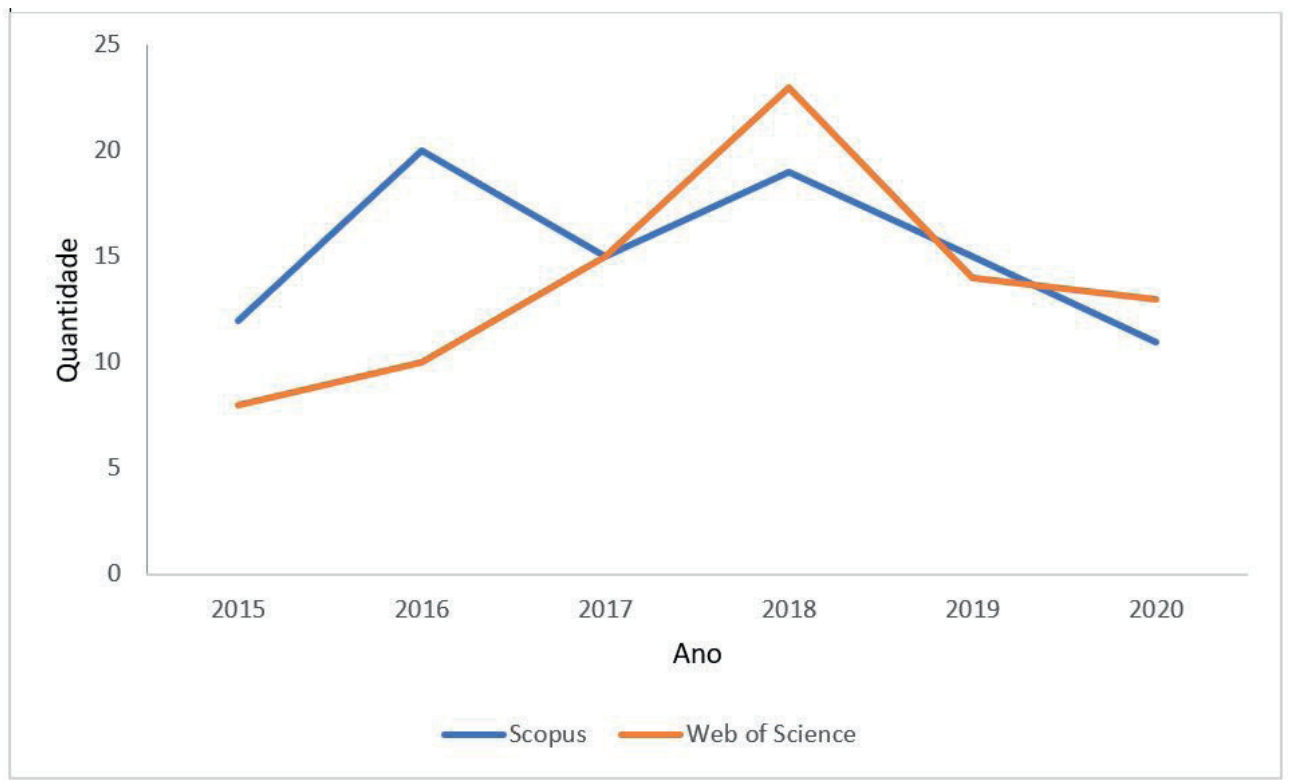

Fonte: elaboração própria (2020).

Dos documentos categorizados nas buscas, apresenta-se, no gráfico 2, a tipologia obtida nas duas bases, os do tipo: artigo de conferência e artigo científico com destaque. Foram encontrados na Web of Science para os do tipo conferência, 40 artigos, e, na Scopus, 38. Para os artigos periódicos, 37 na Web of Science e 33 na Scopus, sendo considerados os mais escolhidos por seus autores, fato que se explica devido à agilidade para participação em eventos específicos e na publicação como os de conferências, e pela garantia de revisões para os do tipo periódico, os quais são submetidos, não dependendo de uma janela fixa de submissão.

Gráfico 2 - Tipos de documentos

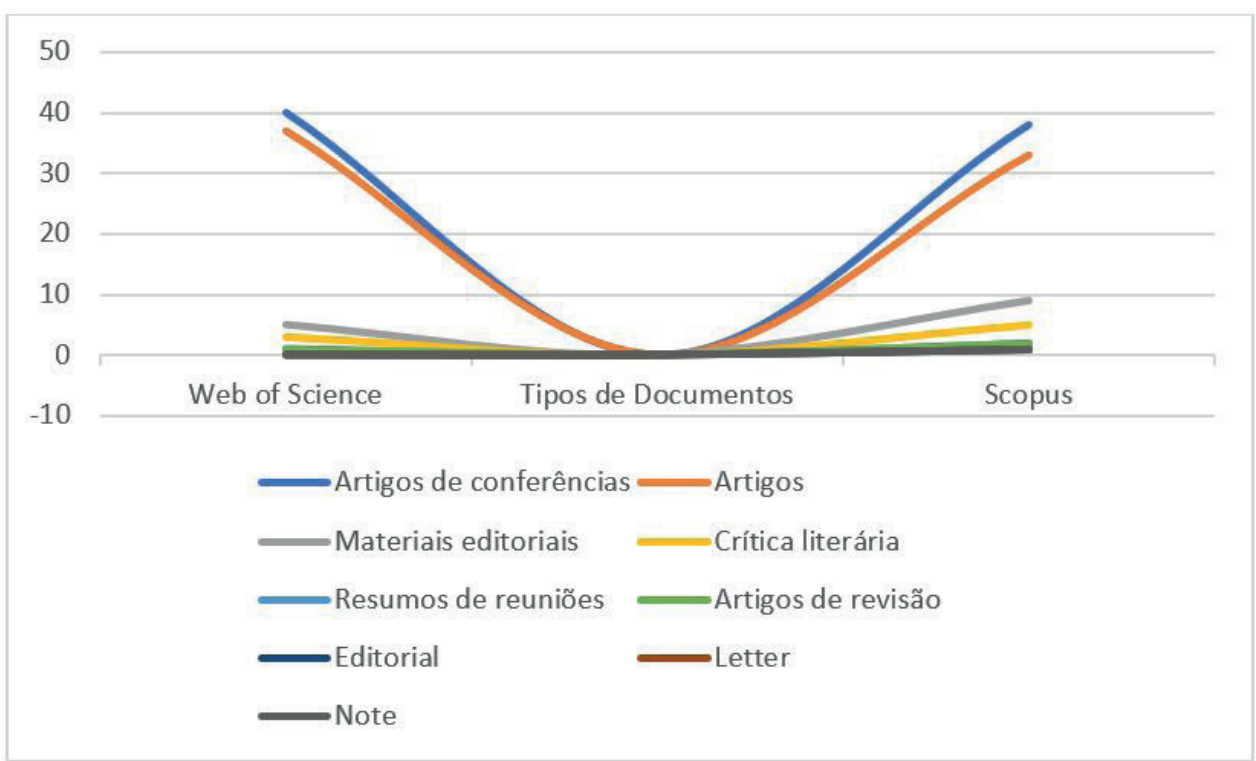

Fonte: elaboração própria (2020). 
Ao focar nos dados que indicaram quais as áreas de conhecimento estudaram o design thinking para geração de inovação, entre os documentos obtidos na base Scopus, representados no gráfico 3, destacaram-se: agricultura, ciências biológicas, artes e humanidades. Com esses dados, notou-se que as temáticas são estudadas em diversas áreas consideradas distantes como as sociais e as exatas.

Gráfico 3 - Publicações por áreas de conhecimento

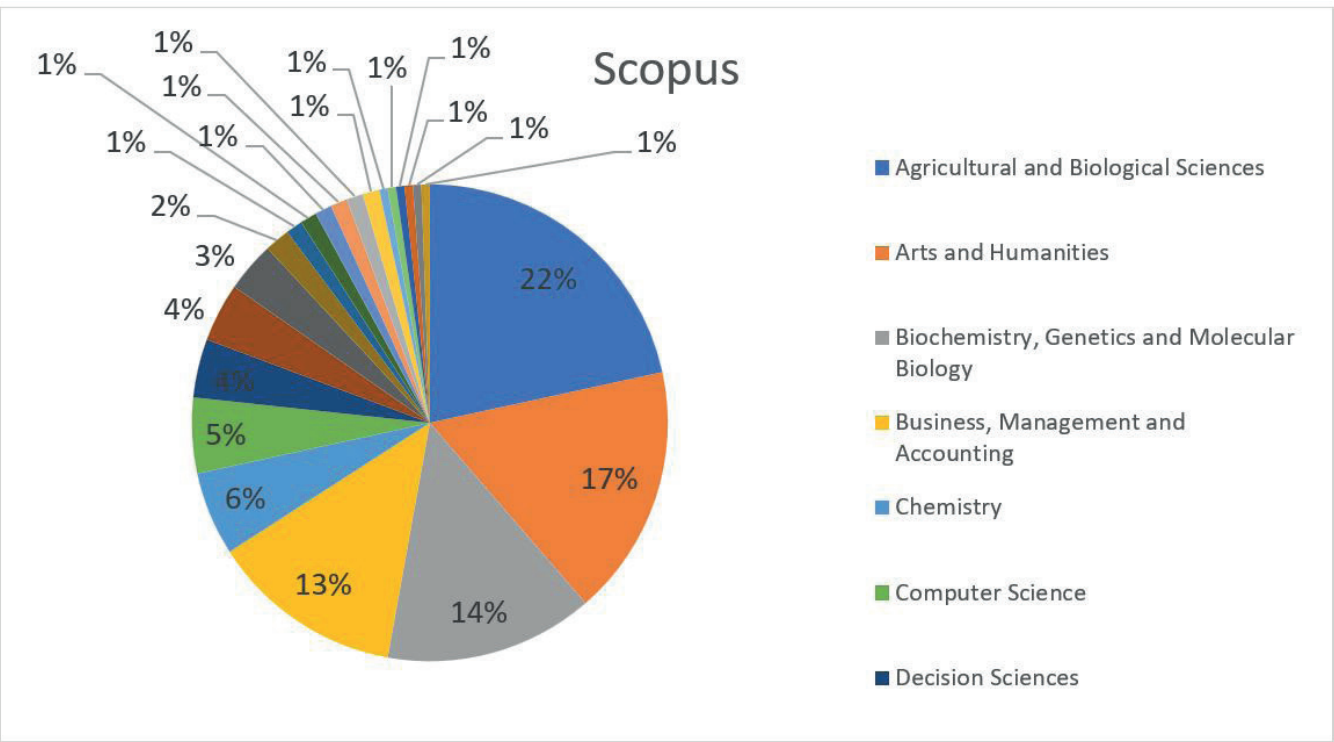

Fonte: elaboração própria (2020).

Continuando a discussão dos resultados por áreas de conhecimentos, destacaram-se na base Web of Science: educação, engenharia e economia como as que possem maior número de documentos, evidenciando o caráter multidisciplinar dessas temáticas estudadas (gráfico 4).

Gráfico 4 - Publicações por áreas de conhecimento

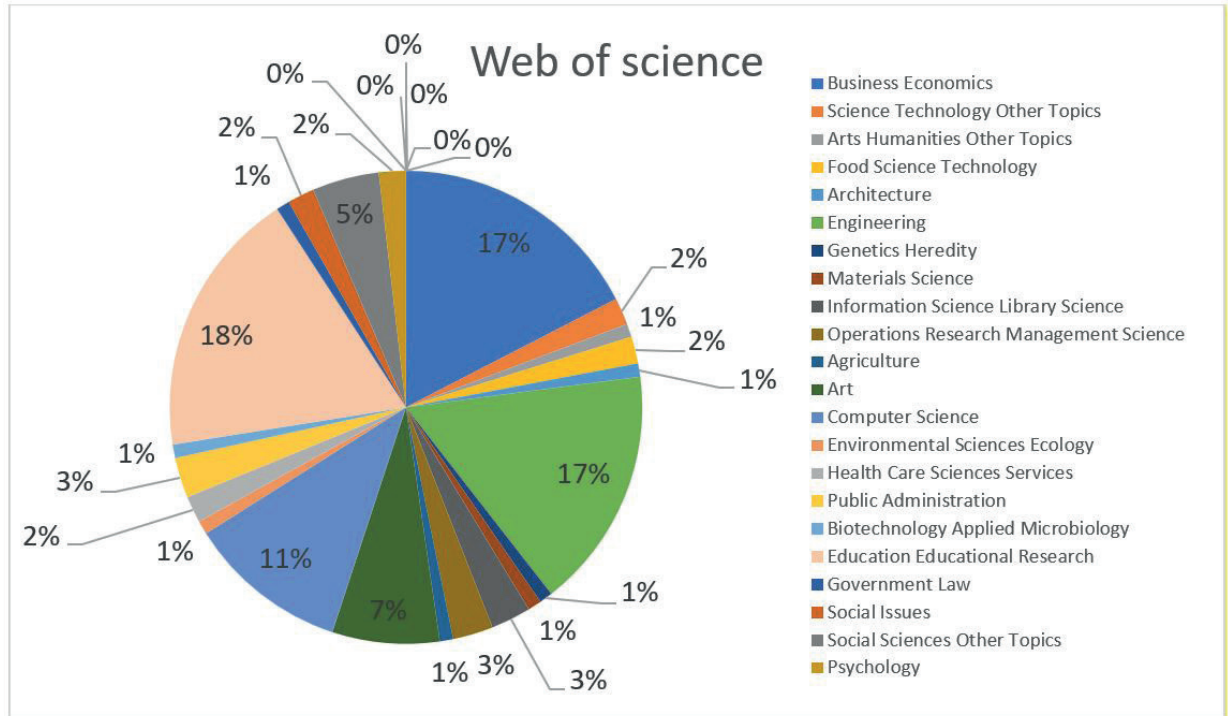

Fonte: elaboração própria (2020). 
As publicações nestas áreas de conhecimento reforçaram o que Vianna et al. (2012) afirmaram: que a inovação orientada pelo design complementa a visão do mercado de que, para inovar, é preciso focar no desenvolvimento ou na integração de novas tecnologias e na abertura e/ou atendimento a novos mercados. Com o DT, a arte se junta a ciência e a tecnologia para desenvolver novas soluções para as organizações (VIANNA et al., 2012).

No gráfico 5 , ao analisar as publicações por instituições, as que se destacaram nas bases estudadas foram: 1 . University of Cape Town localizada na cidade do Cabo - África do Sul), caracterizada por ser uma instituição de ensino superior pública, mais antiga do país e considerada a de melhor qualidade na África; 2. A
Universidade de Oslo que faz parte da Rede de Universidades das Capitais da Europa (UNICA), partícipe de uma organização que envolve 24 universidades e que dá uma ênfase particular a políticas no âmbito de temáticas urbanas e de gestão universitária; 3 . Universidade Stanford é uma universidade de pesquisa privada situada em Palo Alto, Califórnia, Estados Unidos, e uma das instituições mais prestigiadas do mundo, com a maior seletividade de graduação, e a posição de primeira colocada em várias pesquisas e medições no país; 4. Babson College - é uma escola particular de negócios em Wellesley, Massachusetts. Fundada em 1919, seu foco central é a educação para o empreendedorismo (GOOGLE, 2020).

Gráfico 5 - Publicações por Instituição de Ensino e Pesquisa

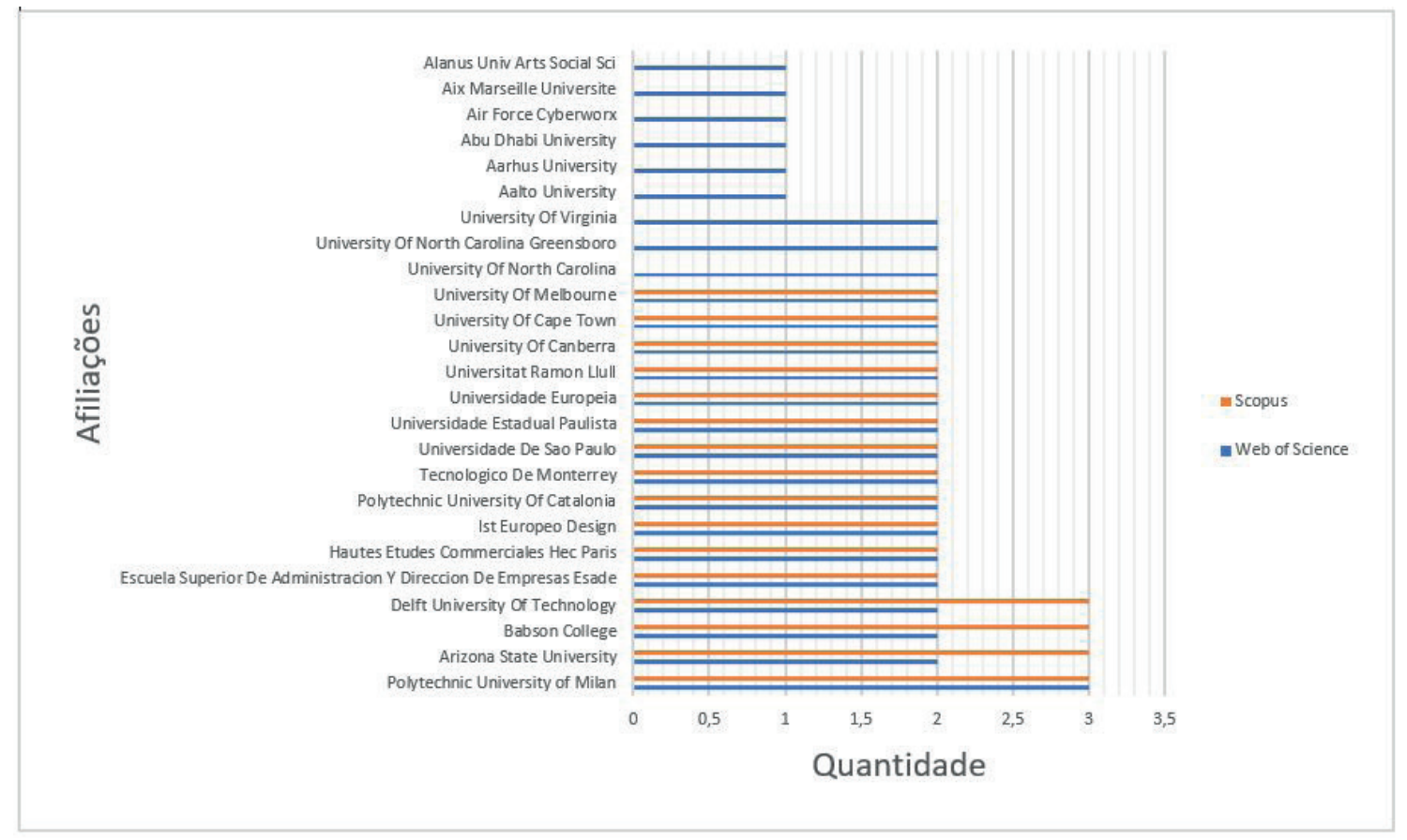

Fonte: elaboração própria (2020).

Um indicador importante na bibliometria é o quantitativo por país, como os expostos no gráfico 6, nos quais se observou que os Estados Unidos se sobressaíram com 22\% das publicações em face dos demais países. Embora exista uma diversidade de países, a língua predominante foi o inglês, presente em $96 \%$ das publicações e $2 \%$ distribuídos entre espanhol e francês. 
Gráfico 6 - Publicações por países

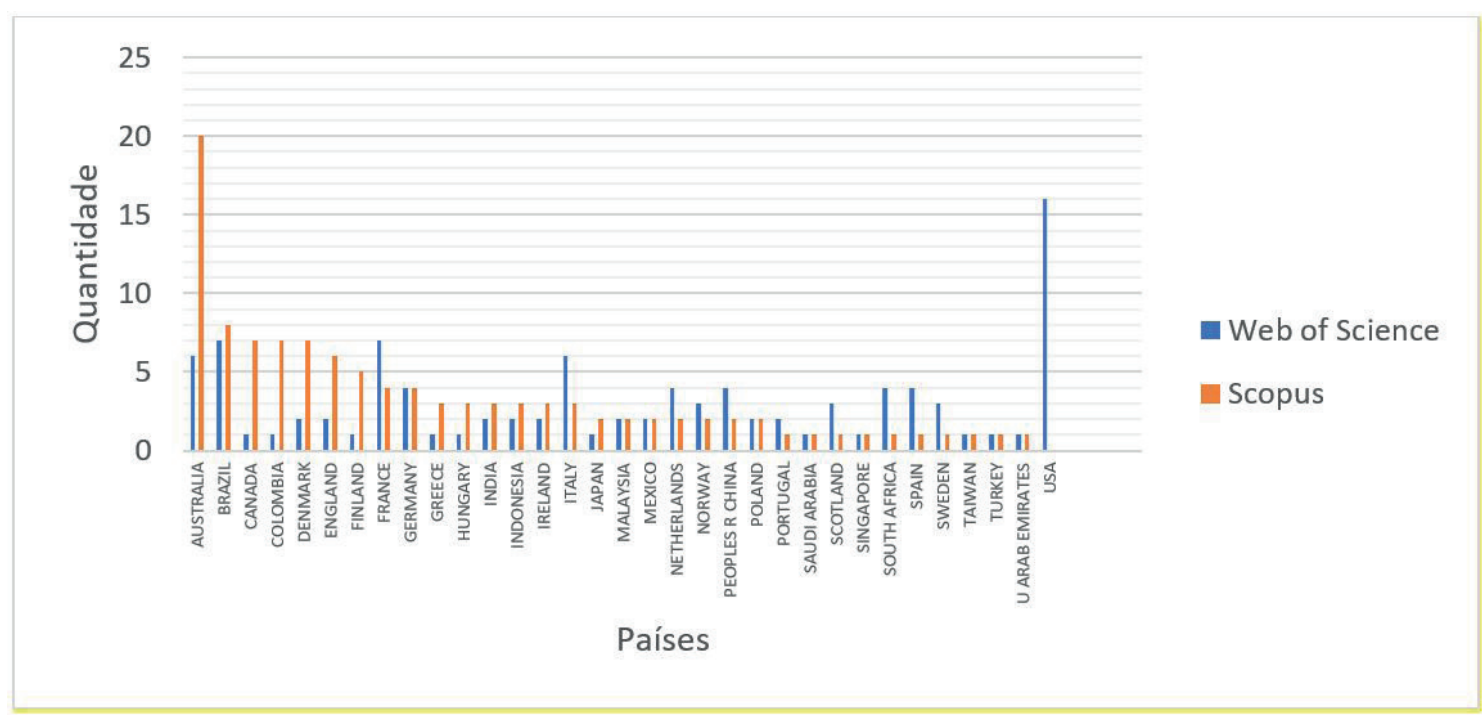

Fonte: elaboração própria (2020).

Os dados do quadro 01 tratam das publicações por periódico ou evento. Entre os mais procurados ou com uma maior quantidade de publicação, estão os seguintes: design jornal, Lecture Notes In Computer Science e ACM International Conference Proceeding Series, na Base Scopus. Os de destaque na base Web of Science são documentos que foram submetidos em eventos, como os evidenciados no quadro 1 na área de inovação, 6 documentos, e outro na de pesquisa em educação, 3 documentos, dados justificados devido à diversidade de documentos e eventos categorizados na base aos quais abordaram as temáticas deste estudo.

Quadro 1 - Publicações por Periódicos ou Eventos

\begin{tabular}{|l|c|l|c|}
\hline \multicolumn{1}{|c|}{ Periódicos ou Eventos } & $\begin{array}{c}\text { Web of } \\
\text { Science }\end{array}$ & \multicolumn{1}{|c|}{ Periódicos ou Eventos } & Scopus \\
\hline $\begin{array}{l}\text { 10th International Conference Of Education } \\
\text { Research And Innovation Iceri2017 }\end{array}$ & 6 & Design Journal & 5 \\
\hline $\begin{array}{l}\text { 11th International Conference Of Education } \\
\text { Research And Innovation Iceri2018 }\end{array}$ & 3 & $\begin{array}{l}\text { Lecture Notes In Computer Science } \\
\text { Including Subseries Lecture Notes } \\
\text { In Artificial Intelligence And Lecture } \\
\text { Notes In Bioinformatics }\end{array}$ & 4 \\
\hline $\begin{array}{l}\text { 12th International Conference Of Education } \\
\text { Research And Innovation Iceri 2019 }\end{array}$ & 2 & $\begin{array}{l}\text { ACM International Conference } \\
\text { Proceeding Series }\end{array}$ & 3 \\
\hline $\begin{array}{l}\text { 12th International Technology Education And } \\
\text { Development Conference Inted }\end{array}$ & 2 & $\begin{array}{l}\text { Advances In Intelligent Systems And } \\
\text { Computing }\end{array}$ & 2 \\
\hline $\begin{array}{l}\text { 13th International Technology Education And } \\
\text { Development Conference Inted2019 }\end{array}$ & 2 & $\begin{array}{l}\text { Design Thinking New Product } \\
\text { Development Essentials From The } \\
\text { Pdma }\end{array}$ & 2 \\
\hline $\begin{array}{l}2016 \text { Ieee Acm International Workshop On } \\
\text { Software Engineering In Healthcare Systems Sehs }\end{array}$ & 2 & $\begin{array}{l}\text { Design Thinking Research Making } \\
\text { Design Thinking Foundational }\end{array}$ & 2 \\
\hline $\begin{array}{l}2017 \text { Ieee International Professional } \\
\text { Communication Conference Procomm }\end{array}$ & 2 & Healthcare & 2 \\
\hline $\begin{array}{l}2018 \quad \text { Xiii Technologies Applied To } \\
\text { Electronics Teaching Conference Taee }\end{array}$ & 2 & Interaction Design And Architecture S & 2 \\
\hline
\end{tabular}




\begin{tabular}{|l|c|l|c|}
\hline $\begin{array}{l}2019 \text { 7th International Forum On Industrial } \\
\text { Design }\end{array}$ & 2 & $\begin{array}{l}\text { International Journal Of Engineering } \\
\text { Education }\end{array}$ & 2 \\
\hline $\begin{array}{l}2020 \text { 11th Ieee International Conference On } \\
\text { Cognitive Infocommunications Coginfocom 2020 }\end{array}$ & 2 & $\begin{array}{l}\text { Proceedings Of The European } \\
\text { Conference On Innovation And } \\
\text { Entrepreneurship Ecie }\end{array}$ & 2 \\
\hline $\begin{array}{l}\text { 20th International Scientific Conference } \\
\text { Economics And Management 2015 Icem 2015 }\end{array}$ & 2 & Project Management Journal & 2 \\
\hline $\begin{array}{l}\text { 26th International Conference On Computers } \\
\text { In Education Icce 2018 }\end{array}$ & 1 & Research Technology Management & 2 \\
\hline $\begin{array}{l}\text { 4th International Conference On Education } \\
\text { And Social Sciences Intcess 2017 }\end{array}$ & 1 & $\begin{array}{l}\text { 2018 Siged International Conference } \\
\text { On Information Systems Education } \\
\text { And Research }\end{array}$ & 1 \\
\hline $\begin{array}{l}\text { 4th International Conference On Higher } \\
\text { Education Advances Head 18 }\end{array}$ & 1 & $\begin{array}{l}\text { 4th International Conference On } \\
\text { Design Creativity Icdc 2016 }\end{array}$ & 1 \\
\hline $\begin{array}{l}\text { Advances In Civil Engineering And Science } \\
\text { Technology }\end{array}$ & 1 & $\begin{array}{l}\text { Achi 2015 8th International } \\
\text { Conference On Advances In Computer } \\
\text { Human Interactions }\end{array}$ & 1 \\
\hline
\end{tabular}

Fonte: elaboração própria (2020).

Observando o quadro 2 e analisando apenas o número de autores com mais publicações, destacaram-se Culén, A.L. e Fixson, S.K., com (3) cada, havendo uma distribuição de 2 ou 1 publicação entre os demais autores, na base Scopus. Ao analisar as autorias e coautorias existentes em cada publicação, em que foi considerado puramente o quantitativo destes, constatou-se um total de 242 entre autores e coautores, havendo 01 publicação com 14 autores. Na base Web of Science, foram evidenciados os autores que mais publicaram; destacam-se com dois documentos cada, tendo mais de dez nesta condição, alguns outros autores aparecem na autoria, contabilizando apenas 01 documento cada, não sendo citados neste quadro, porém eles se destacaram como autores mais citados ver figura 8 .

Quadro 2 - Quantitativo de Publicações por autor

\begin{tabular}{|l|c|l|c|}
\hline \multicolumn{1}{|c|}{ Autores } & Scopus & \multicolumn{1}{c|}{ Autores } & Web of Science \\
\hline Culén A.l. & 3 & Ben Mahmoud-jouini S & 2 \\
\hline Fixson S.K. & 3 & Blomkamp E & 2 \\
\hline Blomkamp E. & 2 & Bragos R & 2 \\
\hline Bourgeois-bougrine S. & 2 & Lande M & 2 \\
\hline Conrad N. & 2 & Charosky G & 2 \\
\hline Douglas T.S. & 2 & Lewis JM & 2 \\
\hline Gasparini A. A. & 2 & Leveratto L & 2 \\
\hline Lande M. & 2 & Mcgann M & 2 \\
\hline Latorre S. & 2 & Conrad N & 2 \\
\hline Lewis J.M. & 2 & Culen AL & 2 \\
\hline Mcgann M. & 2 & Douglas TS & 2 \\
\hline Mourey F. & 2 & Hassi L & 2 \\
\hline Mutsvangwa T. & 2 & Kleinsmann M & 2 \\
\hline Pieniazek M. & 2 & Mutsvangwa T & 2 \\
\hline Uebernickel F. & 2 & Papageorgiou K & \\
\hline
\end{tabular}

Fonte: elaboração própria (2020). 
O destaque por quantidade de citações pode ser observado no gráfico 7 , os mais citados são Blomkamp E., e Lewis, J e McGann, M, na base Scopus, e todos possuem dois documentos publicados correlacionando as temáticas.

Gráfico 7 - Citações por autor

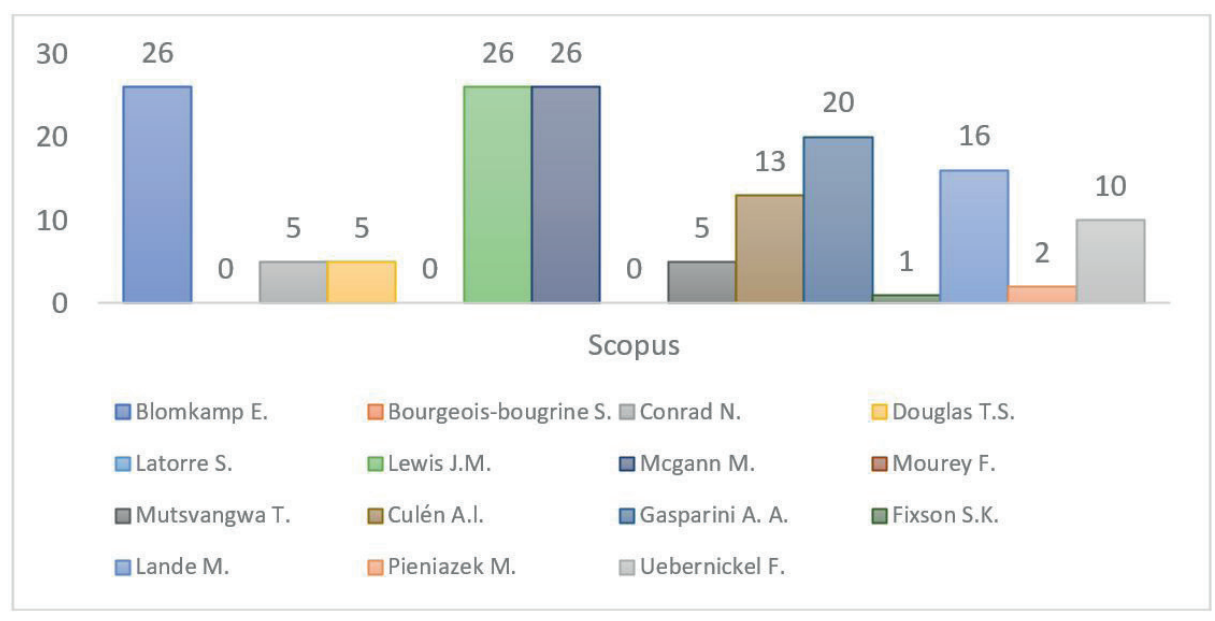

Fonte: elaboração própria (2020).

Considerando que, na base Web of Science, alguns autores tiveram um número maior de citações, destacamos os dois mais citados: primeiramente como autora principal mais citada destaca-se Liedtka, Jeanne, único documento do tipo artigo contido na base, reunindo os dois termos pesquisados, intitulado: Perspectiva: Vinculando Design Thinking com resultados de inovação por meio da redução do preconceito cognitivo, ao qual foi citada, 118 vezes, ao longo do período de estudo deste artigo gráfico 8. Roberts, Jess $P$.; foi o segundo mais citado, contabilizou 87 citações no período, de um único documento intitulado: uma estrutura de design thinking para inovação e gestão de saúde, publicado em 2016.

Gráfico 8 - Citações por autor

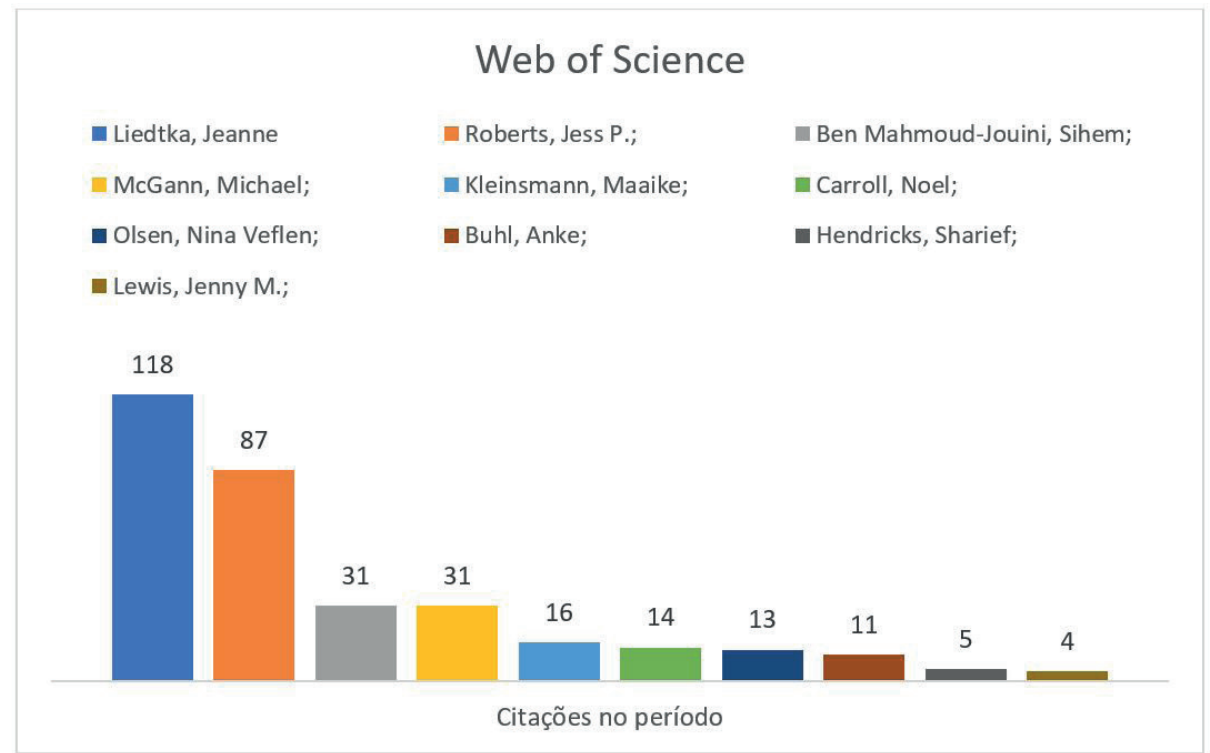

Fonte: elaboração própria (2020). 
Retomando o objetivo do artigo que foi apresentar a relação entre Inovação e metodologias para despertar o criativo, como a ferramenta design thinking que atua como facilitadora para gestão empreendedora da inovação e atrelando as palavras chave de maior intensidade, obtidas na base de dados Scopus, de acordo com o aplicativo de análise de conteúdo VOsviewer, observou-se, na figura 2 que os termos centrais desses documentos são os que também se destacam na amostra analisada, fator importante que pode oportunizar estudos futuros seguindo uma abordagem qualitativa.

Figura 2 - Palavras-chave

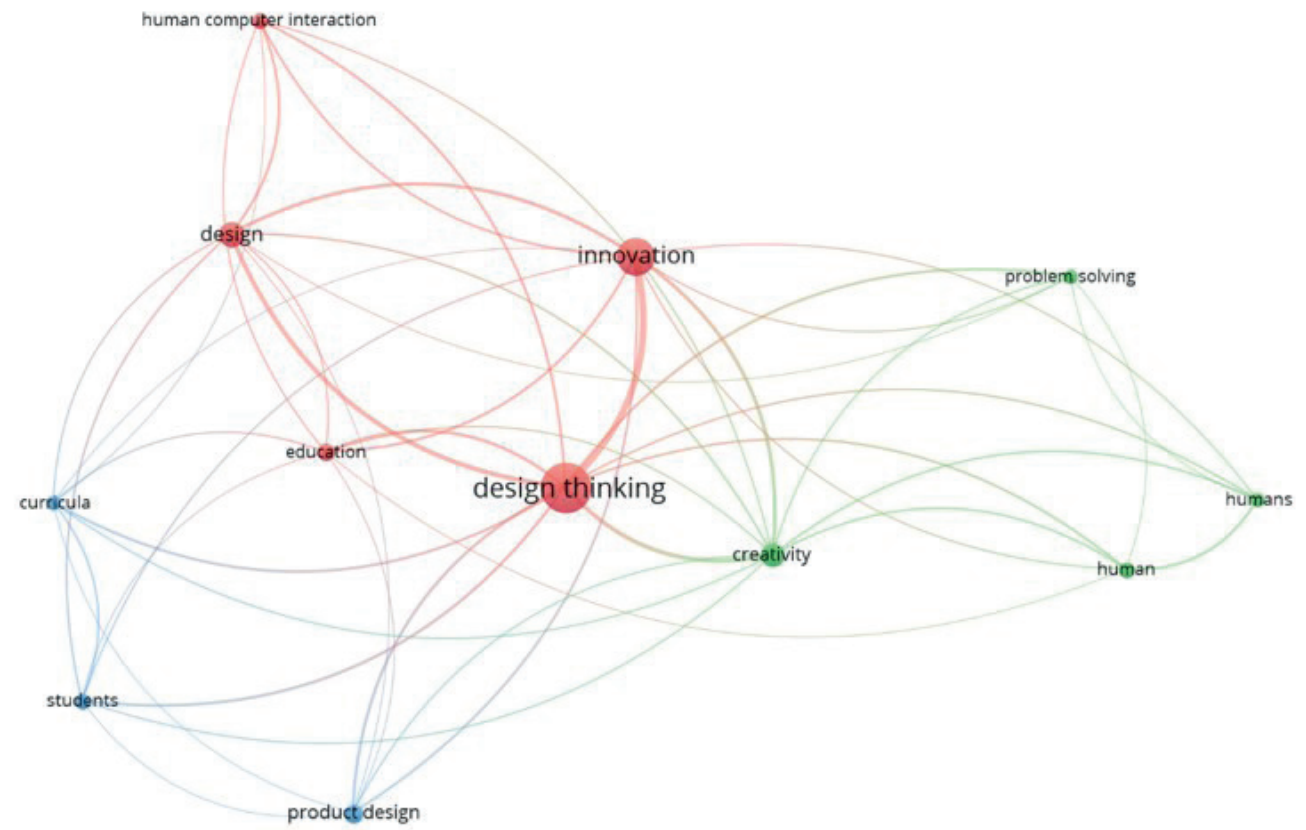

Fonte: elaboração própria (2020).

Alguns outros resultados poderiam ter sido incluídos neste estudo com a mesma abordagem quantitativa, como idioma, tipo de acesso, editoras, grupos de pesquisa; porém, considerando a limitação do ensaio, optou-se pela escolha dos centrais que qualificam as temáticas, pois a metodologia do design thinking é abrangente tanto na diversificação de áreas como de abordagens, considerando que esta visa buscar novos caminhos para a inovação.

\section{CONSIDERAÇÕES FINAIS}

Diante dos resultados apresentados ao longo da discussão, podemos observar que, com a globalização, a inovação se apresenta como um campo importante e deve estar presente em diversas áreas do conhecimento. A multidisci- plinariedade de conhecimento, demonstrada no estudo, destacou-se pela diversidade de instituições e periódicos, e também os anos de 2016 e 2018, com maiores números de publicações, destacando os Estados Unidos com a maior participação nas publicações. Observou-se, também, uma maior concentração de publicações, envolvendo a participação de dois autores, com $29 \%$ do total, enquanto o menor percentual foi de 1\% relativo a uma produção com 14 autores.

Quanto às bases estudadas, ressaltamos que as duas são de caráter multidisciplinar, possuem algumas diferenças quanto ao agrupamento dos dados e tratamento, mas ambas possibilitam a obtenção de dados na mesma categoria, fator que facilita a realização de estudos comparativos; além da possibilidade da utilização de softwares de comparação de dados 
existentes. Nas duas bases, são confeccionados gráficos que facilitam na leitura dos dados, como também transferências em diversos formatos, como xls, e para outros tipos de ferramentas de análise de dados.

Por fim, Inovação e Design Thinking destacadas no estudo como principais palavras-chave, quando associadas a outros termos como educação e criatividade, abrem possibilidades de estudo da geração inovação com base em uma visão sistêmica, e focado na contribuição do DT para solução de problemas por meio de desafios encontrados dentro das diversas áreas de conhecimento.

Portanto, ao findar esta pesquisa, acredita-se que outros aspectos podem ser examinados com maior amplitude, como áreas da saúde e abrangendo novas bases como fonte de consulta e comparação de dados para elaboração de trabalhos futuros sobre as temáticas.

\section{REFERÊNCIAS}

ALENCAR, E. M. L. S. A gerência da criatividade: abrindo as janelas para a criatividade pessoal e nas organizações. São Paulo: Makron Books, 1996.

BONINI, L. A.; SBRAGIA, R. O Modelo de Design Thinking como Indutor da Inovação nas Empresas: Um Estudo Empírico. Revista de Gestão e Projetos, v. 2, n. 1, p. 3-25, 2011.

BROWN, T.; WYATT, J. Design thinking for social innovation. Development Outreach, v. 12, n. 1, p. 29-43, 2010.

CONSIDINE, M. Thinking outside the box? Applying design theory to public policies. Politics \& Policy, v. 40, n. 4, p. 704-724, 2012.

DORST, K. The core of design thinking and its application. Design Studies, v. 32, n. 6, p. 521532, 2011. doi: 10.1016 / j.destud.2011.07.006.

GORDON, S. et al. Improving the front end of innovation with information technology. Research-Technology Management, v. 51, n. 3, p. 50-58, 2008.
HACKLIN, F. et al. Core rigidities in the innovation process: a structured benchmark on knowledge management challenges. International Journal of Technology Management, v. 45, n. 3, p. 244-266, 2009.

KOEN, P. et al. Providing clarity and a common language to the" fuzzy front end". Research-Technology Management, v. 44, n. 2, p. 46-55, 2001.

LIEDTKA, J. Perspective: linking design thinking with innovation outcomes through cognitive bias reduction. Journal of Product Innovation Management, v. 32, n. 6, p. 925-938, 2015.

OECD. Oslo Manual 2018: Guidelines for Collecting, Reporting and Using Data on Innovation. 4th ed. The Measurement of Scientific, Technological and Innovation Activities. Paris/ Eurostat, Luxembourg: OECD Publishing, 2018.

PÄRTTÖ, M.; SAARILUOMA, P. Explaining failures in innovative thought processes in engineering design. Procedia-Social and Behavioral Sciences, v. 41, p. 442-449, 2012.

SCHUMPETER, J. A. O processo de destruição criadora. Capitalismo, socialismo e democracia. Rio de Janeiro: Zahar, 1984.

SHARIF, N.; BAARK, E.; LAU, A. K. W. Innovation activities, sources of innovation and R\&D cooperation: evidence from firms in Hong Kong and Guangdong Province, China. Journal Technology Management, v. 59, n. 3-4, p. 203-234, 2012.

TOHIDI, H.; JABBARI, M. M. Different stages of innovation process. Procedia Technology, v. 1, p. 574-578, 2012.

VERGANTI, R.; ÖBERG, Å. Interpreting and envisioning: a hermeneutic framework to look at radical innovation of meanings. Industrial Marketing Management, v. 42, n. 1, p. 86-95, 2013.

VIANNA, Mauricio et al. Design Thinking: inovação em negócios. Rio de Janeiro: MJV Press, 2012. 